\title{
Características Ecosistémicas asociadas a la actividad ganadera en Arauca (Colombia): Desafíos frente al cambio climático
}

\author{
Ecosystem characteristics associated with livestock farming \\ in the Arauca department (Colombia): challenges regarding \\ climate change
}

\section{Características dos ecossistemas associadas à pecuaria em Arauca (Colômbia): Desafios da Mudança Climática}

\author{
Carolina Ramos-Montaño ${ }^{1}$; Mary R. García-Conde ${ }^{2}$
}

1 Bióloga, MSc; Laboratorio de Manejo Integrado de Ecosistemas y Biodiversidad, Escuela de Ciencias Biológicas,
Universidad Pedagógica y Tecnológica de Colombia, Tunja, Colombia.
2 Bióloga, PhD. Departamento de Biología, Universidad Nacional de Colombia, Bogotá, Colombia.
Email: carolina.ramos@uptc.edu.co

Recibido: enero 17 de 2013

Aceptado: febrero 19 de 2015

\begin{abstract}
Resumen
En la llanura inundable de la Orinoquía colombiana (departamento de Arauca) se realizó la caracterización de cuatro tipos ecosistémicos con diferentes grados de intervención por ganadería, durante la intensa temporada seca de enero a marzo 2010: sabana de pastizal, sabana arbolada, bosque de galería y bosque conservado del piedemonte. En cada uno de ellos se evaluaron las condiciones microclimáticas (humedad y temperatura del aire), las características físico químicos del suelo (capacidad de retención de agua, capacidad de intercambio catiónico, pH, contenido de fósforo y de micronutrientes), la diversidad vegetal (riqueza y abundancia) y el potencial regenerativo (diversidad y porcentaje de germinación del banco de semillas del suelo), con el fin de establecer cuáles parámetros ecosistémicos son los más afectados por la ganadería durante la sequía, y evaluar la capacidad de regulación ambiental y el potencial de recuperación natural. Los resultados demostraron que en casi todos los aspectos el tipo ecosistémico de pastizal es ampliamente contrastante con relación a los bosques conservados del piedemonte, siendo estos últimos los lugares con mayor biodiversidad, menor variabilidad en temperatura y humedad relativa, mayor capacidad de retención de agua, mayor variabilidad topográfica que brinda sitios específicos de regeneración y contenidos más balanceados de micronutrientes en el suelo. Los bancos de semillas, que albergan propágulos que soportan la temporada seca para germinar en condiciones más favorables, fueron más diversos y abundantes en la sabana arbolada, mostrando que este ecosistema tiene mayores opciones de recuperación ecológica que la sabana de pastizal. El estudio demuestra la importancia de la cobertura arbórea en la llanura inundable, especialmente en la autorregulación ecosistémica durante temporadas climáticas desfavorables. Se recomienda la implementación de sistemas de producción ganadera que involucren el componente forestal, y la conservación de los remanentes de bosque.
\end{abstract}

Palabras clave: Bosque de galería, bosque del piedemonte, Orinoquía, pastizal, regulación climática, sabana arbolada, suelo. 


\begin{abstract}
Four types of ecosystem having different degrees of livestock intervention were characterised regarding the Colombian Orinoquia region's floodplain (Arauca department) during an intense dry season lasting from January to March 2010: savannah pastureland, wooded savannah, gallery forest and piedmont conserved forest. Each ecosystem's microclimatic conditions (humidity and air temperature), the soil's physical-chemical characteristics (water retention capacity, cation exchange capacity, $\mathrm{pH}$, phosphorous and micronutrient content), vegetal diversity (richness and abundance) and regenerative potential (soil seed bank diversity and germination rates) were evaluated for establishing which ecosystem parameters were most affected by livestock during the drought and evaluating environmental regulation capacity and natural recuperation potential. The results demonstrated that (regarding almost all aspects) the pastureland-type ecosystem contrasted greatly with piedmont conserved forest, the latter having the greatest biodiversity, least variability concerning temperature and relative humidity, greatest water retention capacity, greatest topographical variability providing specific regeneration sites and the most balanced micronutrient content in soil. The seed banks, harbouring propagules enduring the dry season so as to germinate in more favourable conditions, had the greatest diversity and abundance in wooded savannah, thereby highlighting the fact that this ecosystem had the greatest options for ecological recuperation than savannah pastureland. The study showed the importance of tree cover on the floodplain, especially regarding ecosystem self-regulation during seasons involving unfavourable weather. Livestock production systems involving a forestry component are recommended, as well as ensuring that forest remnants are conserved.
\end{abstract}

Key words: Gallery forest, piedmont forest, Orinoquía, pastureland, climate regulation, wooded savannah, soil.

\title{
Resumo
}

Na várzea da região do Orinoco da Colômbia (departamento de Arauca) foi realizada a Caracterização de quatro tipos de ecossistemas com diferentes graus de intervenção por pecuária, durante a estação seca intensa no período de janeiro a março de 2010: Pastagem de cerrado, savana arborizada, mata de galeria e da floresta preservada do piedemonte. Em cada ecossistema foram avaliadas as condições microclimáticas (humidade e temperatura do ar), as características físicas e químicas do solo (capacidade de retenção de água, capacidade de troca catiônica, pH, teor de fósforo e micronutrientes), diversidade vegetal (riqueza e abundância) e o potencial regenerativo (diversidade e porcentagem de germinação do banco de sementes do solo), a fim de estabelecer quais dos parâmetros do ecossistema são os mais prejudicados pelo gado durante a seca, e avaliar a capacidade de regulação ambiental e potencial de recuperação natural. Os resultados mostraram que, em quase todos os aspectos do tipo de ecossistema de pastagem é amplamente contrastante em relação às florestas preservadas do piedemonte, sendo o último dos lugares de maior biodiversidade, menor variabilidade na temperatura e humidade relativa, maior capacidade de retenção de água, maior variabilidade topográfico que oferece sítios específicos de regeneração e conteúdo equilibrado de micronutrientes no solo. Os bancos de sementes, propágulos que suportam a estação seca para germinar em condições mais favoráveis, foram mais diversos e abundantes na savana arborizada, mostrando que este ecossistema tem maiores opções de recuperação ecológica que a savana de pastagem. O estudo demonstra a importância da cobertura de árvores em área de várzea, especialmente na auto regulação do ecossistema durante as estações meteorológicas desfavoráveis. Recomenda-se a implementação de sistemas de produção de gado que envolve o componente florestal é a conservação dos remanescentes florestais.

Palavras-chave: Mata de galeria, mata de piedemonte, Orinoquia, pastagem, regulação do clima, savana arborizada, do solo.

\section{Introducción}

Se estima que las pasturas para producción animal ocupan cerca del $25 \%$ de la superficie terrestre, han reemplazado un $10 \%$ de los bosques húmedos tropicales, contribuyen a la contaminación hídrica y atmosférica, y al fenómeno de calentamiento global (Meléndez et al., 2005, Steinfeld et al., 2006, FAO 2007, Steinfeld y Wassenaar 2007). La intensificación y aumento del área usada para ganadería en el mundo responde a una población creciente con demandas cada vez mayores de carnes y lácteos para consumo (Asner et al., 2004), causando preocupación especialmente en los países latinoamericanos donde ecosistemas naturales de la Orinoquía y Amazonía podrían ser intervenidos por pasturas.
El suelo es un componente fundamental para la integridad de los procesos ecosistémicos. La capacidad de retención de agua, el desplazamiento de fluidos y la absorción de nutrientes por las plantas están estrechamente relacionados con la agregación y estructura del suelo (Marschner 1995, Amèzketa 1999, Eweis et al., 1999). El impacto de la actividad ganadera puede traer serios efectos a largo plazo en sus propiedades físicas y químicas. De acuerdo con Sadeghian et al., (1998) y Drewry et al., (2008), son numerosas las evidencias de que la compactación de los primeros $15 \mathrm{~cm}$ del suelo, la disminución general de la porosidad, la insuficiencia del sistema hídrico suelo-planta-aire, la reducción de la mesofauna benéfica, los desbalances en los aportes de nutrientes y la limitación del crecimiento radical, son fenómenos asociables a la ganadería intensiva. Todos 
estos efectos llevan en conjunto a una pérdida considerable en la fertilidad del suelo.

Actualmente entre el 22 y $27 \%$ de la ganadería del país se desarrolla en la Orinoquía (Encuesta Nacional Agropecuaria 2008; FEDEGAN 2009; Benavides-Estévez 2010); pero en forma paralela al éxito de esta actividad, se estima que la región sufrió una deforestación de cerca de 255 mil hectáreas en el período 20002007 (IDEAM 2010). El uso potencial de los suelos en la región no es exclusivamente ganadero; se considera que más del $20 \%$ de sus suelos tiene vocación forestal o debe destinarse a la conservación (IGAC 2012), atendiendo además la necesidad de proteger la funcionalidad ecosistémica a lo largo de la cuenca del Río Orinoco (Lasso et al., 2010). Específicamente en el departamento de Arauca, se ha determinado que resultado de este conflicto de uso del suelo el $8 \%$ del territorio ha sido sobre-utilizado (IGAC 2012).

La preocupación acerca del impacto de la ganadería sobre los ecosistemas naturales aumenta si se tiene en cuenta que los suelos de la región son superficiales, presentan una estructura débil con baja infiltración, reciben muy bajos aportes de materia orgánica, presentan procesos dominantes de ferralización, con una alta transformación y pérdida de bioelementos que frecuentemente se asocia a una baja capacidad de intercambio catiónico (Amézquita-Calero 1998, Rao et al., 2001, Malagón-Castro 2003). El diagnóstico general indica que su fertilidad es limitada y requieren de prácticas agronómicas especializadas que involucren entre otras cosas, el uso de la vegetación nativa (Cortés 1982, Malagón-Castro 2003) y su asociación simbiótica con hongos de micorriza vesículo-arbuscular (Salamanca-Solís y Silva-Herrera 1998, Cuenca et al., 2007, Fuchs y Haselwandte 2008).

Si bien la interacción entre el clima, la ganadería y las quemas para fijación de nutrientes ha sido documentada en escenarios de sabana (Oesterheld et al., 1999, Armenteras et al. 2011), realmente lo que mejor se conoce en Suramérica a nivel ecosistémico proviene de estudios en Argentina y Venezuela (Hernández-Valencia y López-Hernández 1999, 2002, Lavado et al. 1996, San José et al., 2003, Von Bernard et al., 2007) que exponen que la ganadería trae transformaciones drásticas en la composición y estructura vegetal, y reducciones en la materia orgánica y nitrógeno total del suelo. Sin embargo, no se han asociado estas transformaciones de uso del suelo con cambios ambientales a pequeña escala, que a su vez sirvan para dimensionar los posibles efectos del cambio climático. En la Orinoquía este tema es crucial ya que presenta una temporada larga de sequía durante la cual el funcionamiento ecosistémico es mucho más vulnerable, y las tendencias del cambio climático indican que el noreste suramericano tendrá menor precipitación, más número de días secos al año y un incremento en la temperatura promedio de hasta de $4^{\circ} \mathrm{C}$ (Voss et al., 2002, NOAA 2016).

La región de la Orinoquía condensa en territorio colombiano cerca del $3 \%$ del agua del planeta y moviliza un volumen de $5.4 \times 10^{11} \mathrm{~m}^{3}$ al año (Mejía-Gutiérrez 1981, Nieto et al. 2008). De acuerdo con estudios paleo-ecológicos (Behling y Hooghiemstra 1998, Montoya et al., 2011) las sabanas en la Orinoquía han evolucionado con un importante componente arbóreo, que interactuó con los componentes de pastizal durante todo el holoceno hasta que comenzó la historia de uso reciente. Es esperable que la predominancia actual de pasturas para ganadería venga asociada a cambios de la hidrología, los suelos y el clima local. El reservorio de semillas que permanecen dormantes en el suelo por períodos prolongados de tiempo, serviría a su vez para estimar la capacidad de regeneración vegetal y del potencial para recuperar la diversidad ecosistémica (Montenegro-Salcedo et al., 2006, Luo y Wang 2006, Holsinger y Gottlieb 1991).

La presente investigación evaluó las condiciones microclimáticas, las características físico químicas del suelo, la diversidad vegetal y el potencial regenerativo, en cuatro tipos ecosistémicos con diferente grado de impacto por ganadería, con el fin de establecer cuáles atributos fueron los más afectados por la ganadería durante la sequía del año 2010 en el departamento de Arauca. El 2010 fue uno de los años más cálidos de la historia moderna, que ejemplarizó los impactos de cambio climático con altas temperaturas y un descenso del nivel de agua en la región Orinoquía que no se registraba en más de 100 años (NOAA 2016).

\section{Materiales y métodos}

\section{Área de Estudio}

La fase de campo se realizó durante la época seca, entre febrero y marzo del 2010, en varias fincas en la cercanía a la cabecera municipal de los municipios de Arauca y Tame (Colombia) (Figura 1). Las localidades pertenecen a la subregión de llanura inundable de la Orinoquía y comparten un origen geológico del Plioceno, con rocas sedimentarias continentales (SGC, 2015). Molano-Barrera (1998) describe la vegetación del departamento en 3 tipos paisajísticos: Planicies de pantanales y desbordes, sabanas de desborde y selvas de piedemonte. Los suelos son ultisoles y alfisoles de horizonte argílico, que se desarrollan sobre un abanico 


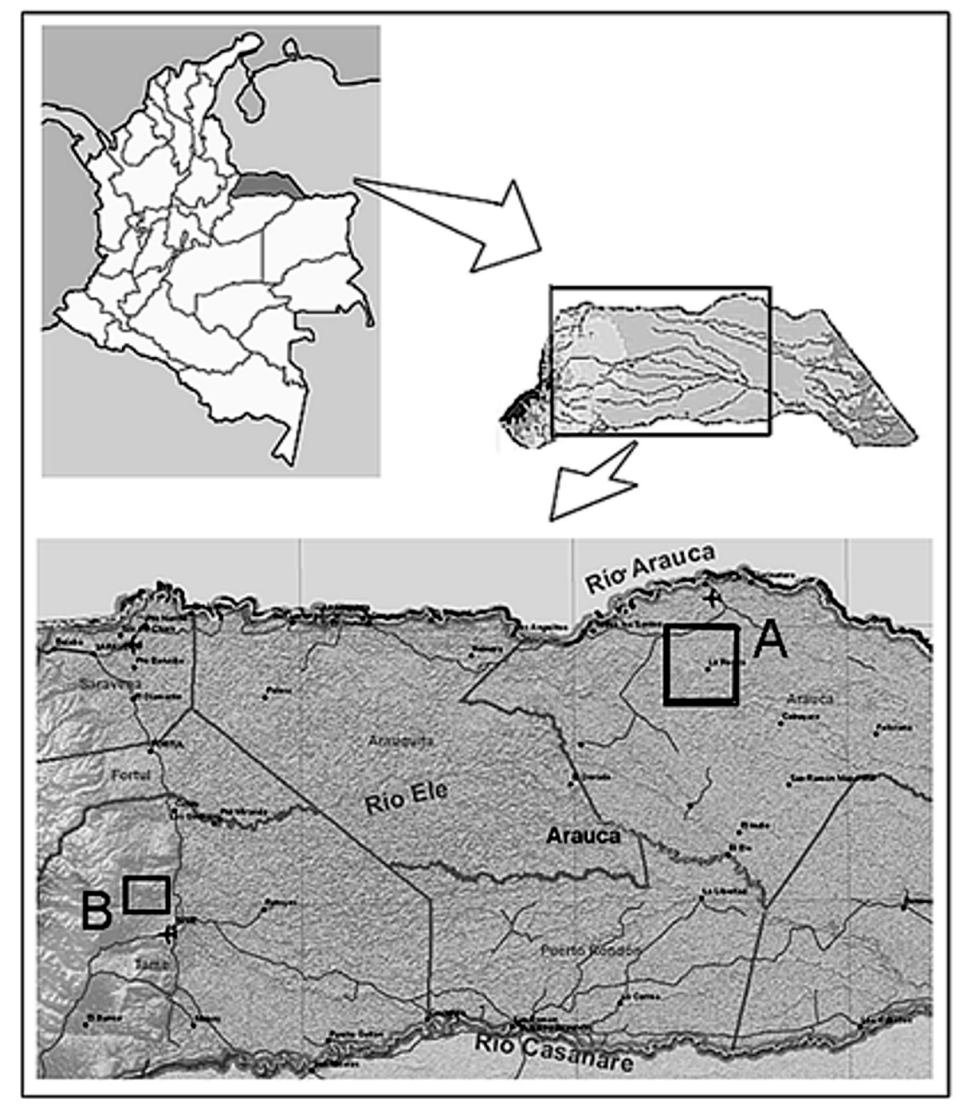

Figura 1. Zona de estudio. A: Fincas la Aguadita y Las Cayenas. B: Finca la Mancha

aluvial (Sarmiento y Pinillos 2001, López 2004). Antecedentes de producción indígena incluyen el uso de plátano, yuca, maíz, frîjoles, arroz, tomate, maní, algodón, tavena, mapuey, ñame, caña, piña, sandía, parsha, lulo, ahuyama, zapallo, ocumo y ajíes (Mejía-Gutiérrez 1998).

\section{Tipos ecosistémicos}

A nivel ecosistémico, el área de estudio albergó cuatro tipos de cobertura:

1) Pastizales (PZ): Cobertura de herbáceas y gramíneas en más de un $95 \%$, con un soporte permanente de ganadería a lo largo del año, talas y quemas recurrentes. Durante la época seca el suelo presenta una denudación que puede superar el $50 \%$ de la superficie. Es el tipo ecosistémico más frecuente en todo el departamento de Arauca. Finca La Aguadita, Arauca $(7.00 .95 \mathrm{~N}-70.39 .95 \mathrm{~W})$.

2) Sabana Arbolada (SA): Dominio de herbáceas y gramíneas en un $75-80 \%$, y presencia de especies arbóreas y arbustivas en forma dispersa en un $20-25 \%$. El pastoreo se da con menos intensidad, especialmente porque corresponden a sectores más inundables durante la época de lluvias. Sin embargo, durante la época seca el suelo presenta una denudación que puede superar el $40 \%$ de la superficie. Finca la Aguadita, Arauca (7.00.94 N - 70.39.95 W).

3) Bosque de Galería (BG): Franja de 20 a 50 m de espesor que protege la ronda de ríos y quebradas, con dominio de especies arbóreas en un 95\% o más. Es notorio el acceso de ganado por la presencia de estiércol en el sotobosque. Finca las Cayenas, Arauca (6.59.14 N - 70.36.43 W).

4) Bosque Continuo del Piedemonte (BC): Remanentes extensos de vegetación nativa y altamente conservados, donde el propietario restringe el acceso de ganado. Ocasionalmente se realizan extracciones para leña. Finca La Mancha, Tame (6.23.72 N - 71.41.89 W).

\section{Caracterización biológica y ambiental de los tipos ecosistémicos}

Riqueza de la Vegetación: En cada uno de los cuatro sitios se trazaron dos transectos de $50 \mathrm{~m}$. y se realizó el conteo de las especies herbáceas, arbóreas y arbus- 
tivas dentro de dos metros a cada lado del mismo. En el caso de gramíneas y plantas clonales la abundancia fue estimada a través del porcentaje en cobertura con que esa especie estaba presente en uno de los dos cuadrantes de $4 \mathrm{~m}^{2}$ cada dos metros.

Microambientes: Para establecer los cambios climáticos se ubicaron 3 termohigrómetros digitales en cada transecto, y se registró la humedad y temperatura a tres horas del día: 7:00-7:30, 12:00-12:30 y 17:0017:30; además de los máximos y mínimos de 24 horas. Además se midió la topografía con un inclinómetro en seis puntos equidistantes de cada transecto.

Propiedades fisicoquímicas del suelo: Se tomaron cinco muestras de suelo por ecosistema para determinar la capacidad de retención de agua (CRA) por método volumétrico. Adicionalmente se extrajeron otras cinco muestras para determinación de la capacidad de intercambio catiónico (CIC), Fósforo total y pH. Finalmente, se tomaron otras cuatro submuestras de suelo en diversos lugares dentro de cada sitio, que fueron en este caso homogenizadas para obtener una muestra donde se determinó el contenido de cuatro micronutrientes: Hierro, zinc, cobre y manganeso.

Capacidad de regeneración: En cinco puntos dentro de cada transecto se colectaron con una pala bloques de $17 \times 17 \mathrm{~cm}$ de superficie y no más de $3 \mathrm{~cm}$ de profundidad, que fueron sometidas en invernadero a condiciones normales de luz y temperatura, y riego controlado. Durante 3 meses se registró el número de semillas germinadas, clasificándolas en morfoespecies.

\section{Análisis estadístico}

Se usaron los software SigmaStat 3.1 y SigmaPlot 9.01 (Systat Software Inc. 2004) para realizar un ANDEVA a dos vías que evaluara la variabilidad de humedad y temperatura debida a la hora del día y a las diferencias ecosistémicas. Los valores extremos (máximos y mínimos), al igual que la topografía fueron analizados a través de un ANDEVA a una vía, para determinar las diferencias ambientales entre tipos ecosistémicos. Se realizó el mismo análisis con las variables fisicoquímicas $\mathrm{CIC}, \mathrm{CRA}, \mathrm{pH}$ y contenido de fósforo. Cuando las variables no cumplieron normalidad $\mathrm{u}$ homogeneidad de varianzas, se procedió al análisis no paramétrico de diferencia por rangos de Kruskal-Wallis. Para establecer las diferencias entre pares de comparación, en cualquiera de los dos análisis, se realizó dos pruebas post-hoc: Tuckey y Student-Newman-Keuls.

Se construyó además una matriz de correlaciones de Spearman con los valores promedio de las diferentes variables fisicoquímicas del suelo, ambientales y biológicas, para encontrar asociaciones entre ellas. Finalmente, se procedió con un análisis de componentes principales para establecer cuáles variables contribuyen en mayor medida a la diferenciación ecosistémica, y con un análisis de factores que halló las asociaciones entre las variables y los factores principales. Todos los análisis multivariados se realizaron con el software Statistica 6.0 (StatSoft Inc. 2001).

\section{Resultados}

La temperatura y la humedad tuvieron una alta variabilidad, con relación a la hora del día y al tipo ecosistémico (Temperatura: $F_{3,20}=110.57 ; p<0.001$. Humedad relativa: $\left.F_{3,20}=87.63 ; p<0.001\right)$ (Figura 2 ). A la hora de mayor estrés climático (12:00-12:30 PM) la temperatura promedio en el pastizal alcanzó un valor de $42^{\circ} \mathrm{C}$, superando ampliamente la temperatura en el bosque continuo $\left(31.3{ }^{\circ} \mathrm{C}\right)$. Igualmente, con relación a la humedad estos sitios fueron contrastantes, con una humedad promedio de $45.67 \%$ en el bosque continuo versus $28 \%$ en el pastizal. El comportamiento ambiental fue más similar entre la sabana arbolada y el bosque continuo, reservando una mayor humedad al comien-
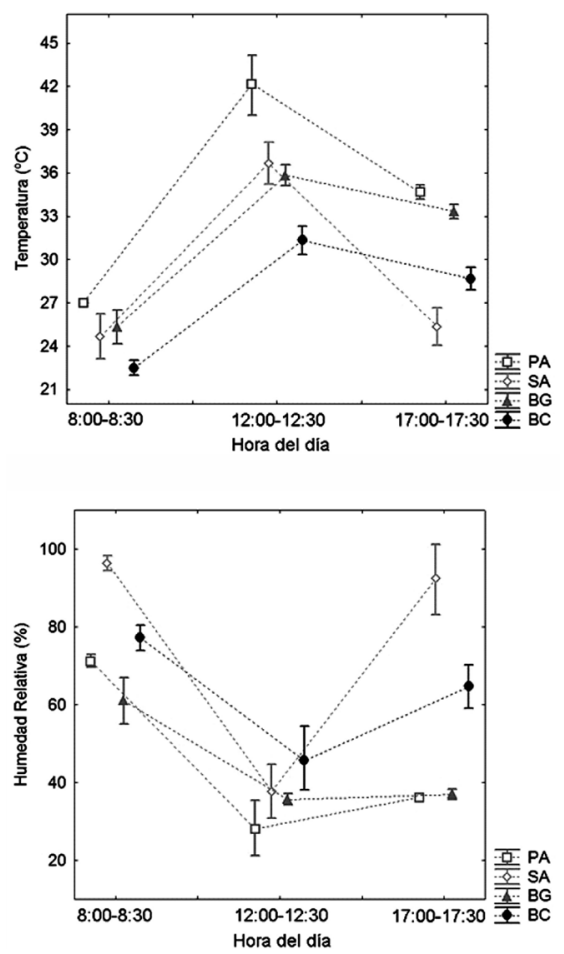

Figura 2. Comportamiento diurno de la temperatura y la humedad relativa (Media \pm Desviación estándar) durante el período seco de Febrero-marzo de 2010, en los cuatro tipos ecosistémicos evaluados. PA: Pastizal; SA: Sabana arbolada; BG: Boque de Galería. BC: Bosque continuo. 
zo del día y manteniendo temperaturas por debajo de $30^{\circ} \mathrm{C}$ al final del día.

Los extremos de temperatura y humedad también fueron significativamente distintos entre tipos ecosistémicos $\left(T^{\circ}\right.$ max: $\mathrm{H}_{3,20}=21.9 ; \mathrm{T}^{\circ}$ min : $\mathrm{H}_{3,20}=20.4$; \%HR max: $\mathrm{H}_{3,20}=18.4 ; \% H R$ min: $\mathrm{F}_{3,20}=25.2 ; p<0.001$ ) (Figura 3). El pastizal tuvo el máximo promedio extremo de temperatura $\left(45.5^{\circ} \mathrm{C}\right)$ y el mínimo promedio extremo de humedad $(17 \%)$, con una variación entre máximas y mínimas que alcanzó $21^{\circ} \mathrm{C}$ y $60 \%$ de humedad. La tendencia general muestra que la diferencia entre máximas y mínimas ambientales disminuye al aumentar el componente arbóreo del ecosistema. La microtopografía es mayor y más heterogénea dentro del bosque de galería y el bosque continuo, con sitios que tienen normalmente más de un 5\% de inclinación. (Figura 4).

Las características fisicoquímicas de los suelos también mostraron diferencias significativas entre tipos ecosistémicos (CRA: $\mathrm{H}_{3,12}=12.8$; pH: $\mathrm{F}_{3,12}=7.32$; CIC: $\mathrm{F}_{3,12}=5.43$; fósforo total: $\mathrm{H}_{3,12}=13.6$; $\mathrm{p}<0.01$ ) (Figura $5)$. El bosque de galería tuvo el mayor promedio de
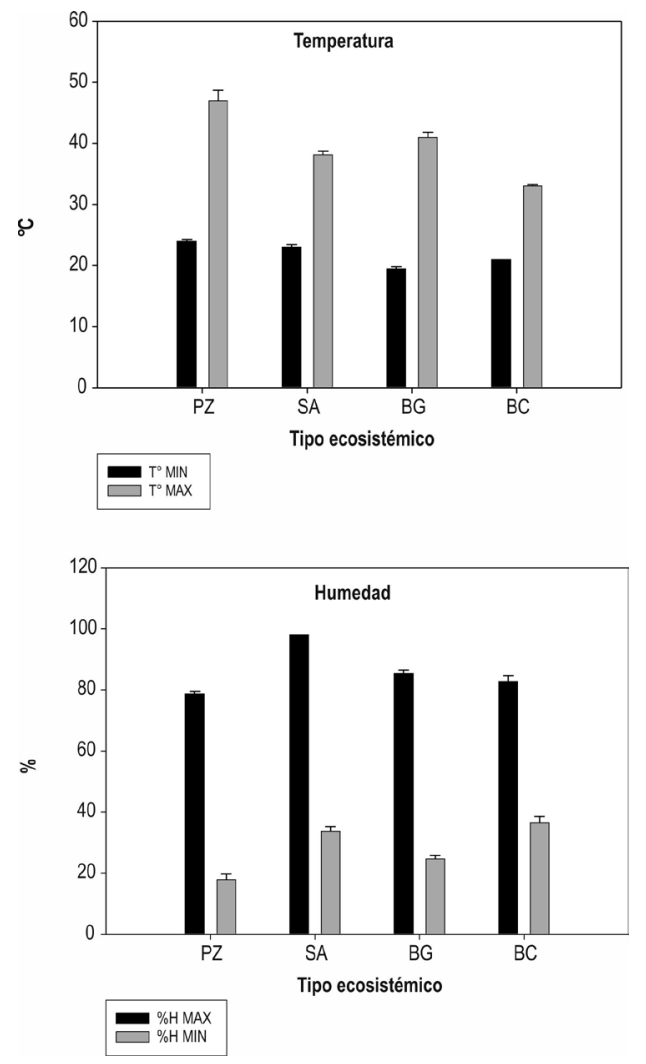

Figura 3. Variabilidad entre los registros máximos y mínimos de temperatura y humedad, en los 4 tipos ecosistémicos. PZ: Pastizal; SA: Sabana Arbolada; BG: Bosque de Galería; BC: Bosque continuo del Piedemonte.

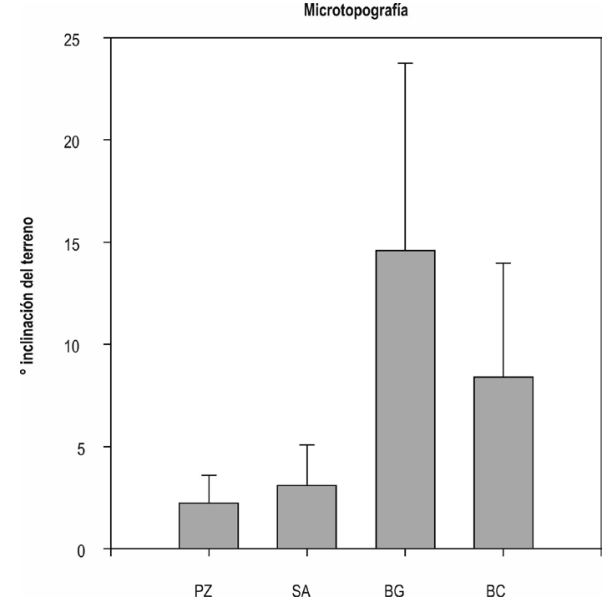

Figura 4. Variabilidad en micro-topografía, evaluada como el grado de inclinación del terreno. PZ: Pastizal; SA: Sabana Arbolada; BG: Bosque de Galería; BC: Bosque continuo del Piedemonte.

CRA (37\%) y un alto promedio de CIC (21 meq/100g). En el pastizal el valor promedio de $\mathrm{pH}$ (4.63) al igual que el de contenido de fósforo $(16.4 \mathrm{mg} / \mathrm{Kg})$ fueron notablemente superiores a los valores promedio para las mismas variables en el bosque continuo $(\mathrm{pH} 4.07 \mathrm{y}$ $1.7 \mathrm{mg}$ de fósforo/ $\mathrm{Kg}$ ). Este ecosistema a su vez tuvo un promedio de CRA (18.8\%) superior al que presenta el pastizal $(11.8 \%)$. La característica del suelo más variable con relación al tipo ecosistémico fue la capacidad de intercambio catiónico, con valores mínimos en la sabana arbolada (8.7 meq/100gr). Los homogenizados provenientes del bosque de galería presentaron altos contenidos de hierro, manganeso y cobre, mientras que el pastizal mostró los más bajos contenidos de hierro, zinc y manganeso. El bosque continuo presentó contenidos balanceados de todos los microelementos evaluados (Tabla 1).

La riqueza de especies arbóreas, al igual que su abundancia, fueron mayores en el bosque continuo (31 especies y 202 individuos), seguido por el bosque de galería (23 especies y 157 individuos). La sabana arbolada tuvo la mayor riqueza total, alcanzando 42 especies leñosas y herbáceas. Este ecosistema además tuvo el mayor número de semillas germinables en sus muestras de suelo $(60 \%)$, a diferencia de los bosques de galería y del bosque continuo ( $5 \%$ del banco germinable en cada caso) (Tabla 2).

Las correlaciones demostraron la relevancia de estos factores: el pH se correlacionó negativamente con la riqueza total (STOT), la riqueza y la abundancia arbórea, mientras que estas dos últimas variables se correlacionaron negativamente con la presencia de fósforo 
en el suelo. El zinc se correlacionó positivamente con la riqueza total. La CIC se correlacionó negativamente con la humedad mínima (\%HMIN). Otras variables que tuvieron poca dirección con relación a los factores 1 y 2, como la inclinación del terreno (INCL) y la capacidad de retención de agua (CRA), estuvieron positivamente correlacionadas. El hierro presentó varias asociaciones importantes con variables ambientales, como una correlación positiva con la topografía y la retención de agua, y una correlación negativa con la temperatura mínima (Figura 6).

El análisis de componentes principales extrajo dos factores que explicaron el $84.2 \%$ de la variabilidad total
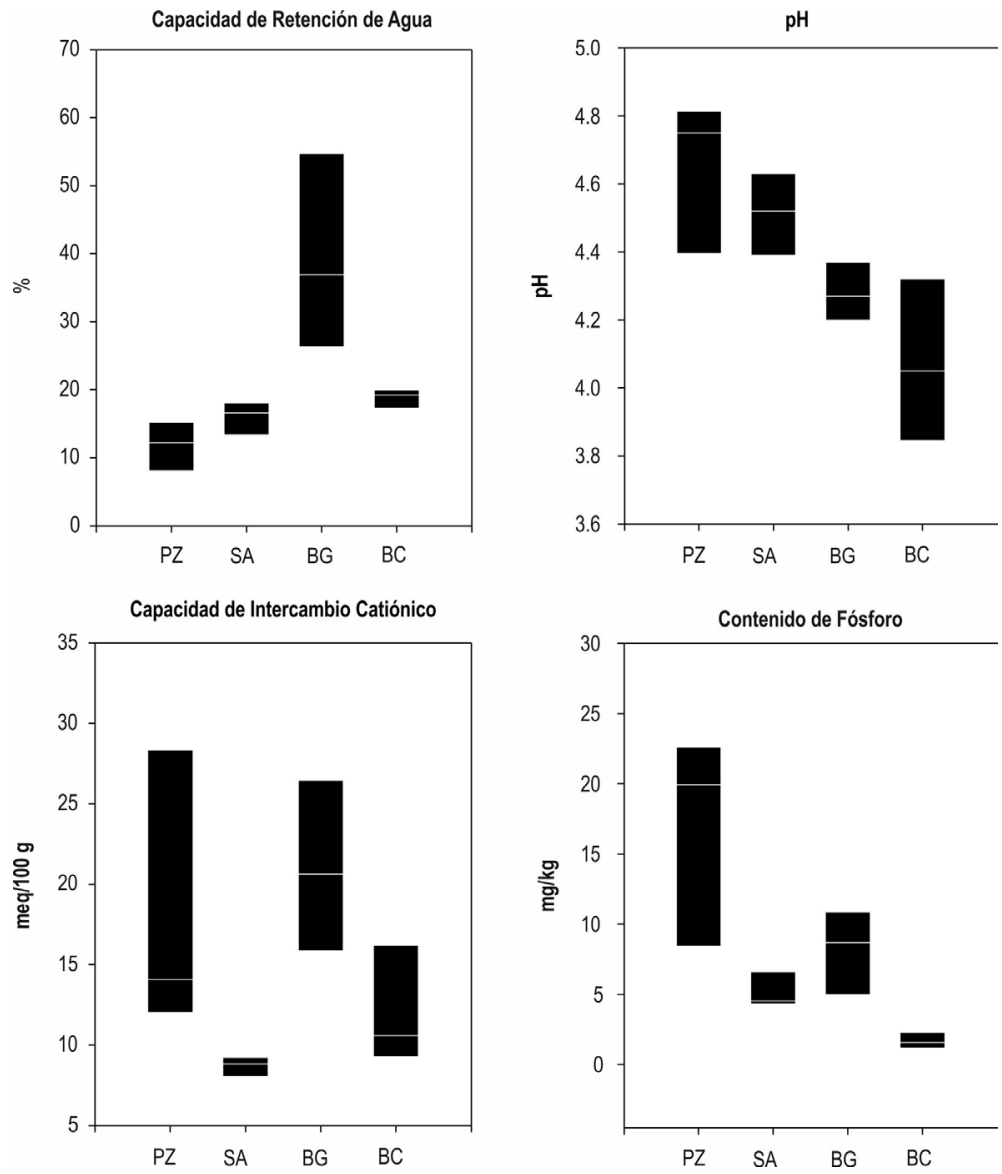

Figura 5. Diferencias entre tipos ecosistémicos, en las características fisicoquímicas del suelo. La línea media representa la mediana, y los límites de las barras corresponden al percentil $90 \%$.

Tabla 1. Contenidos promedio $(\mathrm{mg} / \mathrm{Kg})$ de hierro, cobre, zinc y manganeso en los suelos de cada tipo ecosistémico.

\begin{tabular}{|c|c|c|c|c|c|}
\hline & & $\mathrm{Fe}$ & $\mathrm{Zn}$ & $\mathrm{Cu}$ & $\mathrm{Mn}$ \\
\hline \multicolumn{2}{|l|}{ Pastizal } & 48.9 & 0.38 & 0.26 & 3.06 \\
\hline \multicolumn{2}{|l|}{ Sabana arbolada } & 97.6 & 0.49 & 0.29 & 6.78 \\
\hline \multicolumn{2}{|l|}{ Bosque de galería } & 158 & 0.51 & 0.29 & 7.56 \\
\hline \multicolumn{2}{|l|}{ Bosque continuo } & 115 & 1.36 & 0.24 & 6.24 \\
\hline \multirow{3}{*}{$\begin{array}{l}\text { Niveles críticos } \\
\text { (Molina y Meléndez 2002) }\end{array}$} & Alto & $>50$ & $>10$ & $>20$ & $>50$ \\
\hline & Medio & $5-50$ & $2-10$ & $0.5-20$ & $5-50$ \\
\hline & Bajo & $<5$ & $<2$ & $<0.5$ & $<5$ \\
\hline
\end{tabular}


Tabla 2. Diversidad de la comunidad vegetal y potencial regenerativo en los cuatro tipos ecosistémicos. S: Número de especies. N: Abundancia

\begin{tabular}{|l|c|c|c|c|c|}
\hline \multirow{2}{*}{} & \multicolumn{2}{|c|}{ Leñosas } & \multicolumn{2}{c|}{ Herbáceas } & \multicolumn{2}{c|}{ Banco de Semillas } \\
\cline { 2 - 6 } & S & N & S & S & N \\
\hline Pastizal & 0 & 0 & 21 & 14 & 166 \\
\hline Sabana arbolada & 18 & 86 & 24 & 23 & 331 \\
\hline Bosque de galería & 23 & 157 & 1 & 4 & 28 \\
\hline Bosque continuo & 31 & 202 & 0 & 8 & 28 \\
\hline
\end{tabular}

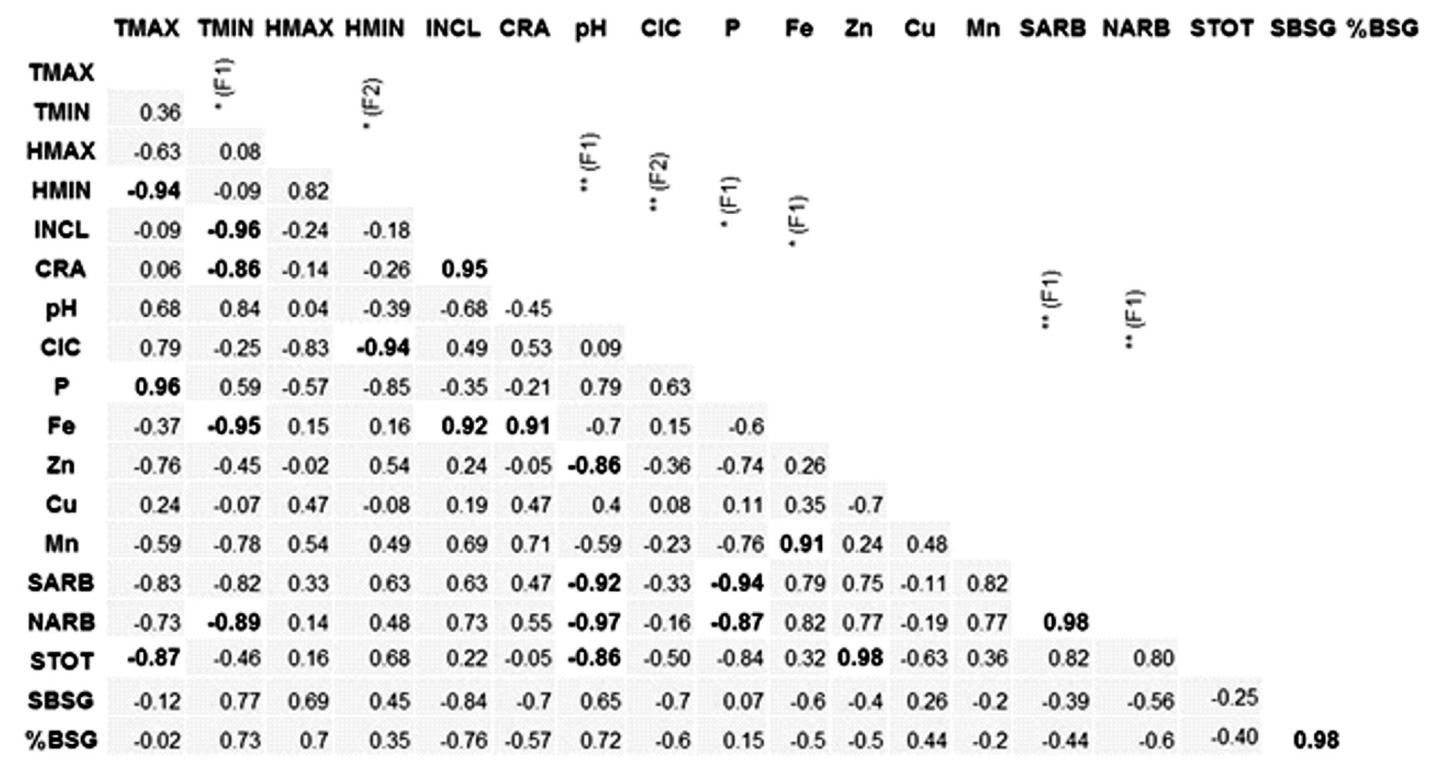

Figura 6. Matriz de correlaciones entre las variables ambientales, fisicoquímicas del suelo y biológicas. Los números en la parte superior de la diagonal corresponden a las variables con valores de alta $(>0.8 ; *)$ y muy alta congruencia $\left(>0.9 ; *^{*}\right)$ con la dirección de los vectores o factores principales 1 y 2 (F1 y F2). INCL: Inclinación del terreno. SARB: Riqueza de especies arbóreas. BSG: Banco de semillas germinable. NARB: Abundancia de arbóreas. STOT: Diversidad total. SBSG: Diversidad banco de semillas germinable. \%BSG: Porcentaje relativo de semillas germinables.

entre ecosistemas. El factor 1 abarcó el $51.7 \%$ de esta variabilidad, y sus componentes principales fueron el $\mathrm{pH}$, la riqueza arbórea (SARB) y la abundancia arbórea (NARB). El segundo factor abarcó el 30.75\% de la variabilidad, y su componente más importante fue la capacidad de intercambio catiónico (Figura 7). Los patrones de asociación multivariante mostraron que todas variables de diversidad biológica (SARB, NARB y STOT) tienden a asociarse con la presencia de micronutrientes como el hierro, manganeso y zinc, caracterizando a los bosques y diferenciándolos del pastizal, donde confluyen una alta temperatura ambiental y una menor acidez del suelo. Por otra parte, las características ambientales que más diferenciaron a la sabana arbolada del bosque de galería fueron la humedad y la CIC.

\section{Discusión}

De acuerdo a Svenson (1996), apenas a mediados del siglo XX el bosque de piedemonte llegó a ocupar hasta un $45 \%$ del departamento de Arauca, siendo reducido a menos de un $10 \%$ mediante procesos extensivos de tala y quema para implementación de ganadería. Los resultados obtenidos en este estudio demuestran una gran diferencia en las características ambientales, pedológicas y biológicas, entre los pastizales que predominan en el paisaje actual y los ecosistemas boscosos. 


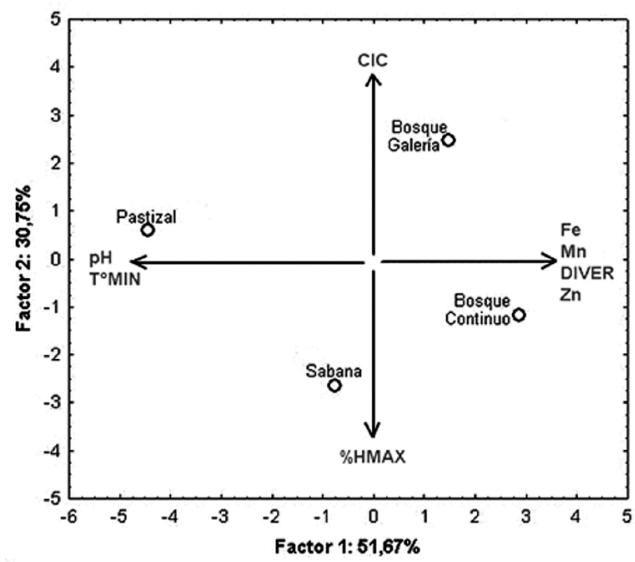

Figura 7. Diferenciación multivariante principal entre los diferentes ecosistemas. DIVER: Diversidad biológica; Incluye SARB, NARB y STOT.

Los pastizales fueron altamente afectados por la sequía, mostrando promedios muy altos de temperatura y muy bajos de humedad durante el día. Durante la realización de esta investigación los termohigrómetros llegaron a registrar temperaturas superiores a $50^{\circ} \mathrm{C}$ en el pastizal a medio día; la época seca se prolongó por casi cuatro meses, y hubo una alta mortalidad de ganado en todo el departamento de Arauca. En contraparte, en el bosque continuo presentó la menor amplitud entre máximas y mínimas de temperatura y humedad, lo que demuestra una alta capacidad de regulación micro-climática durante la época seca. La sabana arbolada fue el tipo ecosistémico climáticamente más parecido al bosque continuo, lo que sugiere que apenas con una participación dispersa del componente arbóreo del 20 al $25 \%$ ya es posible reducir los efectos negativos de la sequía.

En el pastizal además se registró la más baja capacidad de retención de agua y bajos contenidos de microelementos: zinc, cobre y manganeso. Esto trae preocupación en el primer caso por una mayor susceptibilidad de los suelos a la erosión, y en el segundo caso porque el suelo no alcanzaría los requerimientos mínimos para una óptima nutrición bovina (Venuto et al., 2003, Mora et al., 2004, Gupta et al., 2008). Por otra parte, existen evidencias de que los bajos contenidos de micronutrientes en sabanas de pastoreo se relacionan más con la pérdida de estructura del suelo debida a la compactación (Sadeghian et al., 1998) o con la volatilización de nutrientes por quema (Cook 1994). La alta acidez del suelo que se evidenció en toda el área de estudio, limita aún más las deficiencias de estos nutrientes (Taiz y Zeiger 1998, Mora et al., 2004).
Otras características, como altos contenidos de fósforo, alta la capacidad de intercambio catiónico y contenidos balanceados de hierro, fueron características positivas en los suelos de pastizal, en comparación con otros tipos ecosistémicos. El bosque de galería presentó algunas características cercanas con el pastizal, en relación a la capacidad de intercambio catiónico y el contenido de fósforo, pero otras específicas como una máxima capacidad de retención de agua y niveles de hierro demasiado altos, que podrían estar asociados con la ferralización de minerales del suelo.

La heterogeneidad espacial a pequeña escala, medible a través de un estimador topográfico como la inclinación del terreno, fué mayor en bosque continuo y en bosque de galería, favoreciendo la variabilidad de micro-sitios para el establecimiento y crecimiento de especies nativas, y por ende la diversidad. El bosque de galería contó con 23 especies arbóreas y arbustivas, una riqueza importante y no muy divergente del ecosistema de referencia (bosque continuo). En el caso de la sabana arbolada, su potencial biológico es de 23 especies representadas en el banco de semillas. En cualquiera de los dos tipos ecosistémicos, existe un importante recurso biológico para la recuperación del componente arbóreo, en caso de iniciativas de producción más ecológicas (Acero-Duarte 2007).

Las diversas variables bióticas y abióticas incluidas en este estudio mostraron correlaciones relevantes. La diversidad estuvo relacionada directamente con ambientes con baja temperatura, baja concentración de fósforo y alta de zinc. Además confirmó la relación positiva del hierro con la capacidad de intercambio catiónico. Se demostró que existe una la divergencia ecosistémica entre pastizales y otros ecosistemas durante la época seca (Figura 7). Sabana arbolada y bosque de galería se ubicaron a una distancia multivariante más cercana al bosque continuo que al pastizal.

En general se puede decir que si bien los ecosistemas de sabana funcionan como sumideros de carbono, sosteniendo así un sistema productivo de importancia como la ganadería, ese balance de biomasa a largo plazo no está garantizado luego de eventos de disturbio como la tala, la quema y las sequías (San José y Montes 2003, Hernández -Valencia y López-Hernández 2002, Steinfeld y Wassenaar 2007). Si se tiene en cuenta que por efecto del cambio climático en las próximas décadas se esperan sequías más prolongadas e intensas, como la que tuvo lugar en el 2010, entonces los escenarios de sabana arbolada estarían en mejor capacidad de auto-regulación ambiental y biológica, conservando el potencial de uso. Por otra parte, una estrategia de conservación, como la implementa- 
ción de sistemas forestales o silvopastoriles, podría aumentar sustancialmente el secuestro de carbono (San José y Montes 2003), y mantendría la diversidad a nivel regional que se está perdiendo por la dominancia de pastizales.

El calentamiento global, en interacción con las actividades antrópicas, es capaz de ocasionar la transformación ecosistémica, incluso hasta el punto de llegar a la desertización (Dodd 1994). La Orinoquía sería una región vulnerable, teniendo en cuenta que buena parte de la ganadería se realiza en suelos de vocación agrícola y forestal, y los pastizales generan un $31 \%$ de los gases de efecto invernadero en Colombia (MalagónCastro 1998, IDEAM et al. 2015). Es recomendable que entidades públicas y productivas trabajen en conjunto para atender algunas de las siguientes acciones: (1) técnicas eficientes de manejo de ganado, (2) sistemas agro-silvopastoriles, (3) bio-recuperación ecosistémica y (4) conservación de los remanentes de bosque.

\section{Agradecimientos}

Este estudio hace parte del proyecto 2020100135852063 de la Universidad Nacional de Colombia: "Colección y caracterización de propágulos para el mejoramiento de suelos y la conservación biológica en la Orinoquía", y ha sido financiado a través del Instituto de Estudios de la Orinoquía. Agradecemos además la valiosa colaboración del agrónomo Jorge Hernández en el reconocimiento del área de estudio y del ingeniero Francisco Mijares en la identificación del material vegetal. A toda la amable comunidad araucana durante la realización del proyecto.

\section{Referencias}

Acero-Duarte LE. 2007. Plantas útiles de la Cuenca del Orinoco. Corporinoquía - Gobernación de Casanare. Bogotá. 607p

Amézquita-Calero E. 1998. Propiedades físicas de los suelos de los Llanos Orientales y sus requerimientos de labranza. En Romero G, Aristizábal D \& Jaramillo C. (Eds). Encuentro Nacional de labranza de Conservación. Memorias. Villavicencio, Colombia. 145-174.

Amèzketa E. Soil Aggregate Stability: A Review. J Sustain Agric.1999;14(1-2):83-151.

Armenteras D, Retana J, Molowny R, Román RM, González F, Morales $M$. Characterising fire spatial pattern interactions with climate and vegetation in Colombia. Agr Forest Meteorol. 2011;151:279-289

Asner GP, Elmore AJ, Olander LP, Martin RE, Harris AT. Grazing systems, ecosystem responses, and global change. Annu Rev Environ Resour. 2004;29:261-99.

Behling $\mathrm{H}$, Hooghiemstra $\mathrm{H}$. Late Quaternary palaeoecology and palaeoclimatology from pollen records of the savannas of the
Llanos Orientales in Colombia. Palaeogeography, Palaeoclimatology, Palaeoecology. 1998;139(3-4):251 267.

Benavides-Estévez J. 2010. El desarrollo económico de la Orinoquia: Como aprendizaje y construcción de instituciones. In Steiner, R. and V. Traverso (eds.) Colombia 2010-2014: Propuestas de Política Pública. CAF- Fedesarrollo. Pgs. 279-320.

Cook GD. The fate of nutrients durig fires in a tropical savanna. Australian Journal of Ecology, 1994;19(4):359-365.

Cortés LA. 1982. Geografía de los suelos de Colombia. Universidad Jorge Tadeo Lozano. Colombiana de Impresos Ltda. Bogotá $161 \mathrm{pp}$.

Dood JL. Desertification and degradation in Sub-Saharan Africa: The role of livestock. Bioscience. 1994;44(1):359-365.

Drewry JJ, Cameron KC, Buchan GD. Pasture yield and soil physical property responses to soil compaction from treading and grazing: A review. Soil Res. 2008;46(3):237-256. Cuenca G, Cáceres A, Oirdobro G, Hasmy Z, Urdaneta C. Las micorrizas arbusculares como alternativa para una agricultura sustentable en áreas tropicales. Interciencia. 2007;32(1):23-29.

ENA - Encuesta Nacional Agropecuaria. 2008. [consultada en noviembre de 2011] http://www.cci.org.co/oferta/ena/ 2008.

Eweis JB, Ergas SJ, Chang DP, Schroeder ED. 1999. Principios de Biorrecuperación: tratamientos para la descontaminación de suelos y aguas subterráneas mediante procesos biológicos y fisicoquímicos. Mc Graw-Hill, Madrid. 327 p.

FAO - Organización de las Naciones Unidas para la Agricultura. 2007. Ganadería y deforestación. En: Políticas Pecuarias No.3. Subdirección de Información Ganadera y de Análisis y política del sector. Dirección de producción y sanidad Animal. 8p.

FEDEGAN - Federación Nacional de Ganaderos. 2009. Aumenta la productividad de la Ganadería bovina en 7,8\% en los últimos 3 años. Boletín del mes de abril.

Fuchs B, Haselwandte K. 2008. Arbuscular Mycorrhiza of Endangered Plant Species: Potential Impacts on restoration strategies. En: Varma A (Ed.) Mycorrhiza. Springer -Verlag, Berlin - Heidelberg. 565-579.

Gupta UC, Wu K, Liang S. Micronutrients in Soils, Crops, and Livestock. Earth Science Frontiers, 2008;15(5):110-125.

Hernández-Valencia I, López-Hernández D. 1999. Efectos de la quema sobre el ciclo del fósforo en una sabana de Trachypogon. Ecotropicos, 12: 3-7.

Hernández-Valencia I, López-Hernández D. Pérdida de nutrimentos por la quema de la vegetación en una sabana de Trachypogon. Rev Biol Trop. 2002;50(3-4):1013-1019.

Holsinger KE, Gottlieb LD. 1991. Conservation of Rare and Endagered Plants: Principles and Prospects. En: D A. Falk y K E. Holsinger (eds.). Genetics and Conservation of Rare Plants. Oxford University Press, pp.195-208.

IDEAM. 2010. Informe Anual sobre el estado del Medio Ambiente y los recursos naturales Renovables en Colombia - Bosques 2009. Instituto de Hidrología, Meteorología y Estudios Ambientales. Bogotá. 236 pp.

IDEAM, PNUD, MADS, DNP, CANCILLERIA. 2015. Primer Informe Bienal de Actualización de Colombia ante la Convención Marco de las Naciones Unidas sobre el cambio climático. Bogotá. 252pp. 
IGAC. 2012. Estudio de los Conflictos de Uso del Territorio Colombiano. Instituto Geográfico Agustín Codazzi. Bogotá. 211 pp.

Lasso CA, Usma J, Trujillo F y Rial A. 2010. Biodiversidad de la Cuenca del Orinoco: Bases Científicas para la identificación de áreas prioritarias para la conservación y uso sostenible. Alexander Von Humboldt, WWF Colombia, Bogotá. 609p.

Lavado RS, Sierra JO, Hashimoto PN. Impact of grazing on soil nutrients in a Pampean grassland. J Range Manage. 1996;49(5):452-457.

López E. 2004. Aspectos más relevantes de la geomorfología y geología del Piedemonte Llanero de Colombia. Documento del Ministerio de Minas y Energía. Bogotá. 23p.

Marschner H. 1995. Mineral nutrition of higher plants, 2nd edition. Academic Press, Londres. 889 p.

Mora M, Alfaro M, Williams P, Stehr W, Demanet R. Efecto de la aplicación de fertilizantes sobre la acidificación del suelo en relación con el crecimiento y la composición química de una pradera y la producción animal. Rev cienc. suelo nutr veg. 2004;4(1):29-40.

Luo H, Wang K. Soil seed bank and aboveground vegetation within hillslope vegetation restoration sites in Jinshajing hot-dry river valley. Acta Ecologica Sinica. 2006;26(8):2432-2442.

Malagón-Castro D. El recurso suelo en Colombia: Inventario y Problemática. Rev Acad Colomb Cienc Exact Fis Nat. 1998;22:1352.

Malagón-Castro D. 2003 Ensayo sobre Tipología de Suelos Colombianos énfasis en génesis y aspectos ambientales. Rev Acad Colomb Cienc Exact Fis Nat. 2003;27:319-341.

Mejía-Gutiérrez M. 1981. Producción acuática continental colombiana. Documento no publicado, Universidad Nacional de Colombia. Bogotá.

Mejía-Gutiérrez M. 1998. Sistemas de producción en la Orinoquía colombiana. En: C Domínguez (Ed.) Colombia: Orinoco. Fondo FEN Colombia. Bogotá. 271-288.

Meléndez RD, Romero-Cazeaudumec Y, Blavia F. Livestock facilities and pollution of water resources in Venezuela: Current status. Veterinaria Tropical. 2005;29-30(1-2):99-111.

Molano-Barrera J. 1998. Biogeografía de la Orinoquía Colombiana. En: C Domínguez (Ed.) Colombia: Orinoco. Fondo FEN Colombia. Bogotá. 69-101.

Molina E, Meléndez G. 2002. Tabla de interpretación de análisis de suelos. Universidad de Costa Rica, Centro de Investigación Agronómicas. San José.

Montenegro-Salcedo AL, Avila-Parra YA, Mendivelso HO, Vargas O. Potencial del banco de semillas en la regeneración de la vegetación del humedal Jaboque, Bogotá, Colombia. Caldasia. 2006;28(2):285-306

Montoya E, Rull V, Nogué S. Early human occupation and land use changes near the boundary of the Orinoco and the Amazon basins (SE Venezuela): Palynological evidence from El Paují record. 2011;310(3-4):413-426.

Nieto R, Gallego D, Trigo R, Ribera P, Gimeno L. Dynamic identification of moisture sources in the Orinoco basin in equa- torial South America. Journal des Sciences Hydrologiques. 2008;53(3):602-617.

National Oceanic and Atmospheric Administration (NOAA). [consultada en abril de 2016] https://www.ncdc.noaa.gov/sotc/ service/global/significant-extremes/

Oesterheld M, Loreti J, Semmartin M, Paruelo JM. 1999. Grazing, fire, and climate effects in primary productivity of grasslands and savannas. En: LR Walker (Ed.) Ecosystems of disturbed ground. Elsevier. Amsterdam 287-306.

Rao I, Rippstein G, Escobar G, Ricaurte J. 2001. Producción de biomasa vegetal epigea e hipogea en las sabanas nativas. En: G Rippstein, G Escobar, F Motta (Eds.). Agroecología y diversidad de las sabanas de las sabanas en los Llanos Orientales de Colombia. CIAT. Cali. 198-222.

Sadeghian S, Rivera JM, Gómez ME 1998. Impacto de sistemas de ganadería sobre las características físicas, químicas y biológicas de suelos en los Andes colombianos. Memorias de la Conferencia Electrónica de la FAO: "Agroforestería para la Producción Animal en América Latina". Pp.77-95.

San José JJ, Montes RA, Rocha C. Neotropical savanna converted to food cropping and cattle feeding systems: soil carbon and nitrogen changes over 30 years. For Ecol Manage. 2003;184:17-32.

Salamanca-Solis CR. Silva-Herrera MR. 1998. Las micorrizas como alternativa para el manejo Sostenible de los agroecosistemas tropicales. Boletin Técnico No 12. CORPOICA.

Sarmiento G, Pinillos M. Patterns and processes in a seasonally flooded tropical plain: the Apure Llanos, Venezuela. J Biogeogr. 2001;28(8):985-996.

Servicio Geológico Colombiano (SGC). 2015. Mapa Geológico de Colombia a escala 1: 1.000.000. Disponible en http://www2. sgc.gov.co/

Svenson G. 1996. La erradicación de los bosques de la Orinoquía. En: Memorias del Segundo Encuentro de Orinocólogos. CORPES Orinoquía. Bogotá.

Steinfeld H, Gerber P, Wassenaar T, Castel V, Rosales M, de Haan C. 2006. Livestock's Long Shadow: Environmental issues and options. FAO, Roma. 408 p

Steinfeld H, Wassenaar T. The role of Livestock Production in Carbon and Nitrogen Cycles. Annu Rev Environ Resour. 2007;32:271294.

Taiz L, Zeiger E. 1998 Plant Physiology, Sinauer Associates, Inc., Publishers- Sunderland, Massachussets.

Venuto BC, Ward JD, Twidwell EK. Effects of Soil Type and Soil Chemical Composition on Nutrient Content of Annual Ryegrass for Beef and Dairy Cow Nutrition. Journal of Plant Nutrition. 2003;26(9):1789-1799.

Von Bernard H, Vilarino V, Piñeiro G. Theoretical emission of methane in three systems to fatten cattle in Argentina. Cienc Investig Agrar. 2007;34:91-98.

Voss R, May W, Roecknera E. Enhanced Resolution Modelling Study on Anthropogenic Climate Change: Changes in Extremes of the Hydrological Cycle. Int J Climatol. 2002;22(7):755-777. 